\title{
TO STUDY THE EFFICACY OF AUTOLOGOUS PLATELET RICH PLASMA OVER CONVENTIONAL MECHANICAL FIXATION METHODS IN SPLIT THICKNESS SKIN GRAFTING
}

\section{Ankur Deshwali*}

\section{Peeyush}

Kesharwani

\section{Rohan Gupta}

Resident, General Surgery \& Sri Aurobindo medical college \&postgraduate institute, indore,Madhya Pradesh india. ${ }^{*}$ Corresponding Author

Resident, General Surgery \& Sri Aurobindo medical college \&postgraduate institute, indore,Madhya Pradesh india.

Resident, General Surgery \& Sri Aurobindo medical college \&postgraduate institute,indore,Madhya Pradesh india.

ABSTRACT

AIMS AND OBJECTIVES: to determine the efficacy of autologous platelet rich plasma over conventional mechanical fixation methods in split thickness skin grafting.

\section{METHODS :}

- This a prospective randomized controlled study, conducted in the Department of surgery, SAIMS Medical College and P.G Institute, Indore for a period of two years from September 2016 to September 2018. A total of 20 patients were included in the study, and divided into two groups of 10 each randomly. The study was approved by the local ethical committee of our hospital. An informed written consent was obtained from all patients.

- Total of 20 patients were studied; 10 cases were randomly chosen for study with autologous platelet rich plasma and 10 cases received conventional methods like staples/sutures used to anchor the skin grafts in a control group.

RESULTS: Autologous PRP showed faster and better healing rates. With PRP study group instant graft adherence was seen in all cases. Hematoma, graft edema, discharge from graft site, frequency of dressings and duration of stay in hospital were significantly less in the PRP. There were no adverse effects or reactions seen with the use of autologous PRP among the study group. CONCLUSION: The combination of PRP with Split Thickness Skin Graft (STSG) significantly improved clinical outcomes and shortened the wound healing time. Therefore, this treatment combination could provide a way to heal skin after skin graft reconstruction with minimal recovery time. It is found to be highly beneficial in many aspects both to the patient and surgeon based on our results.

KEYWORDS : - platelet rich plasma, Split Thickness Skin Graft (STSG), No cost, faster and better healing rates

INTRODUCTION

Resurfacing of wound beds with split skin graft is the commonest procedure undertaken in the field of plastic surgery. The success of skin graft depends on local vascularity and wound microbiology on one hand and hemostasis and adhesion of skin graft to wound bed on the other. Skin graft is conventionally fixed to wound margins with sutures, staplers, acrylate glue or fibrin glue in order to prevent shearing and seroma under the graft. However, these methods added to the operating time and cost.

Normal platelet counts in blood range from approximately $1,50,000$ to $4,50,000 / \mathrm{cum}^{3}$, whereas platelet rich plasma (PRP) contains platelet concentration above baseline compared to same quantity of whole blood.

Growth factors that are released from platelets in the PRP promote angiogenesis, collagen synthesis and epithelization, reduce dermal scarring and facilitate remodelling STSG healing proceeds through 3 stages which are anchorage, inosculation and maturation. The success of the first two stages is critical to the overall success.

PRP brings about instant adhesion of graft to bed preventing any collection under the graft or undue shear.

PRP provides an immediate surgical hemostatic agent that is biocompatible, safe and effective. It brings about instant adhesion of graft to bed preventing any collection under the graft or undue shear.

PRP accelerates endothelial, epithelial, and epidermal regeneration, stimulates angiogenesis, enhances collagen synthesis, promotes soft tissue healing, decreases dermal scarring and enhances the hemostatic response to injury. primary healing and reduce healing time, likely as a result of shearing force reduction and enhancement of the wound environment with growth factors.

\section{DATA ACQUISITION}

This a prospective randomized controlled study, to test the efficacy of autologous platelet rich plasma in wound beds prior to resurfacing skin grafts instead of conventional methods like sutures, staplers or glue The study was conducted in the Department of General Surgery, SAMC \& PGI , Indore for a period of 6 months from July 2018 to January 2019.

A total of 60 pateints were divided into two groups of 30 each randomly. Detailed history was taken in all cases regarding the duration, mode of onset, progression and associated symptoms.

An informed consent was taken from all the patients. The study was approved by the institutional ethics committee and in line with the declaration of Helsinki and followed the guidelines laid out by the Indian council of medical research (ICMR), Written consent was taken from the patients participating in the study

The etiological factor that might be responsible for chronicity was also elicited

Wound examination was done in all cases

Patients with co morbidities like DM, hypertension were also included in this study.

Following patients were excluded from the study

- Ulcers with evidence of malignancy

- Active infection with pus discharge

- Immune compromised patients

- Indexed ulcer with exposed tendons, ligaments or bone 
METHODS AND MATERIALS

This a prospective randomized controlled study, to test the efficacy of autologous platelet rich plasma in wound beds prior to resurfacing skin grafts instead of conventional methods like sutures, staplers or glue. The study was conducted in the Department of General Surgery, SAMC \& PGI , Indore for a period of 18 months from July 2018 to December 2019. A total of 60 patients were divided into two groups of 30 each randomly. detailed history was taken in all cases regarding the duration, mode of onset, progression and associated symptoms. An informed consent was taken from all the patients. The etiological factor that might be responsible for chronicity was also elicited. Wound examination was done in all cases. Patients with co morbidities like DM, hypertension were also included in this study. Following patients were excluded from the study 1)Ulcers with evidence of malignancy 2)Active infection with pus discharge 3)Immune compromised patients 4)Indexed ulcer with exposed tendons, ligaments or bone.

On the day of surgery, under all aseptic precautions, $10 \mathrm{ml}$ of blood was drawn intravenously from the antecubital region into 2 bulbs containing CPDA $(0.7 \mathrm{ml})$ each. The blood centrifuged at $3000 \mathrm{rpm}$ for $5 \mathrm{mins}$. The supernatant formed is Platelet Poor Plasma (PPP) and buffy coat. PPP and the buffy coat were aspirated and collected in another vacutainer and again centrifuged at 1000 rpms for 5 minutes. The upper half was discarded and the lower half yields concentrated platelet rich plasma.(figure 1.) Approximately $5 \mathrm{ml}$ of $\mathrm{PRP}$ was required for wound area of 100sq.Cm.

After preparation of the wound beds PRP was applied as a thin film over the raw area before application of the graft in case study group. The grafts were assessed for instant adhesion by moving graft with finger and then graft was covered by nonadhesive compressive dressing. In the control group the edge was adequately secured with stapplers or sutures through the graft and surrounding skin. No prp was used in the control group.

As per standard practice first graft inspection and dressing was done on the basis of wetness/soakage of the dressing or $5^{\text {th }}$ day whichever is earlier. Subsequent dressings were changed on the basis of soakage/ wetness of the dressings. Graft success was quantified according to objective parameters used for assessment of efficacy of study like instant adhesion, graft edema, discharge from site, graft loss, day of first graft inspection, frequency of dressing and duration of stay in hospital. The graft loss was considered significant if the patient required secondary grafting for complete wound coverage. The patients were Followed up for a minimum of 1 month post discharge and in some cases upto 3 months.

Stastistical analysis of the data was done using SPSS software (version 21, Chicago, IL, USA). Unpaired student's " $t$ " test and chi-square test were used to find out the statistical significance for $\mathrm{P}<0.05$ was taken as significant

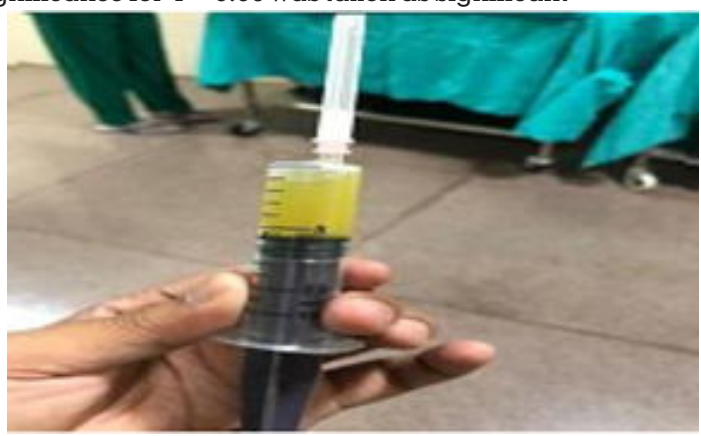

Figure 1: Platelet rich plasma.

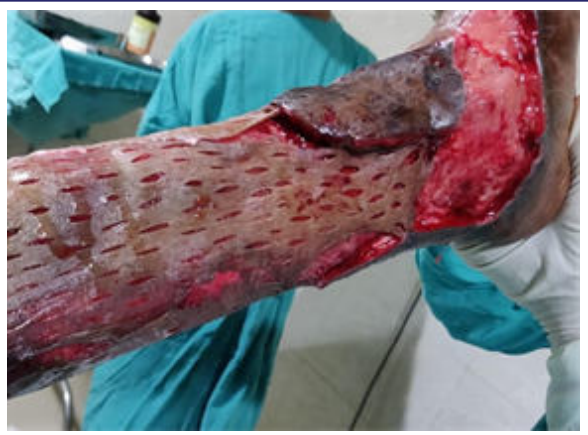

Figure 2

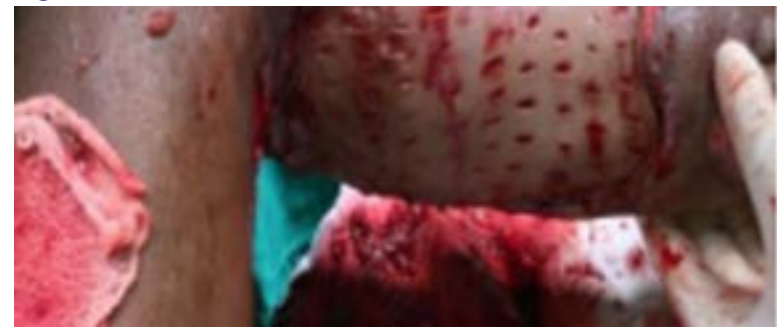

Figure 3

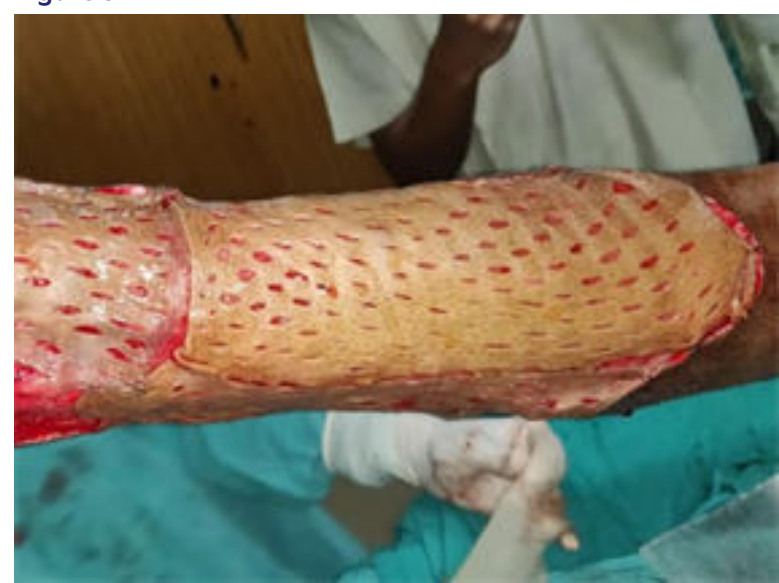

Figure 4

Figure 2,3\&4: Applying platelet rich plasma over the wound bed prior to resurfacing

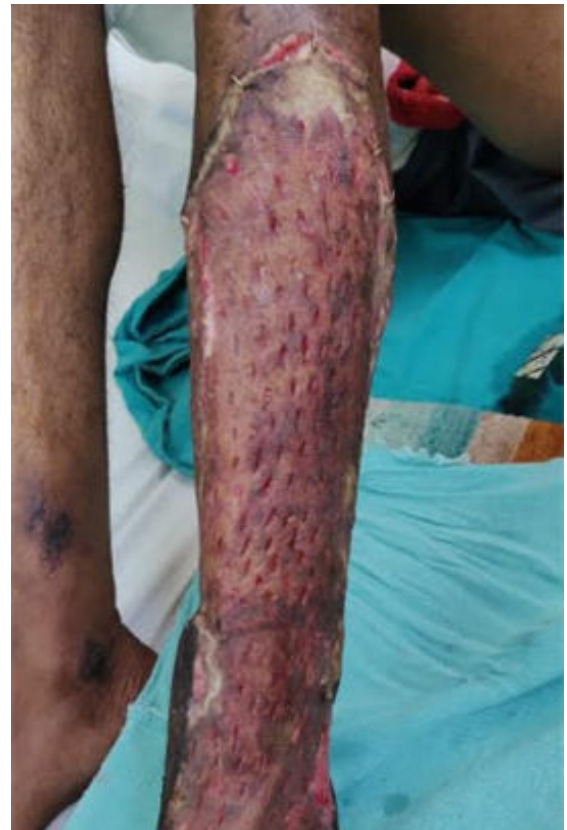

Figure 5 


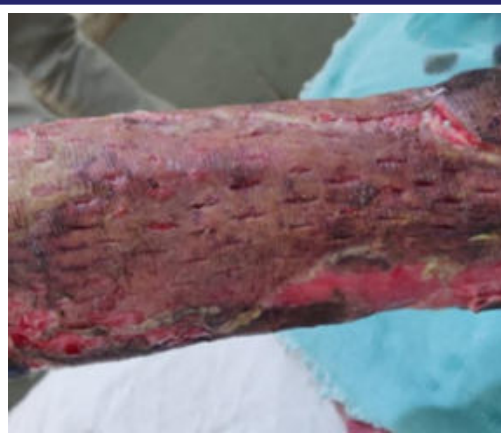

Figure 6

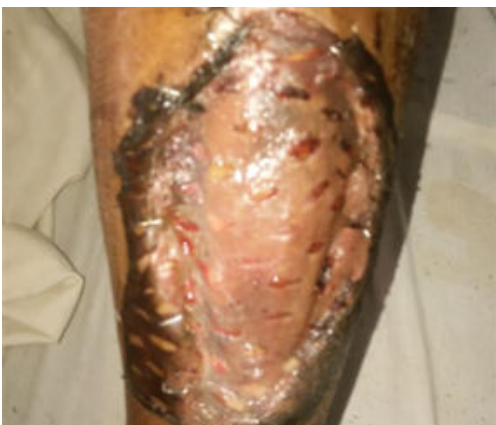

Figure 7

Figure 5,6\&7 Pictures of graft during subsequent dressings

\section{OUTCOMES AND RESULT}

The most common etiology in both the groups was necrotizing fasciitis (13 in case group and 10 in control group), followed by diabetic foot ( 7 in case and 6 in control group) and cellulitis ( 4 in case group and 5 in control group). Other etiologies included burn wound, coverage of amputation stump and wound due to trauma. Among the comorbidities 12 and 10 patients had diabetes in case and control group respectively while 7 and 2 patients had hypertention in case and control group respectively.

All patient's grafts in the case group adhered well within seconds of application compared to $0 \%$ of the control subject. Graft edema with PRP group were present only in $10 \%$ of patients whereas in control group $36.6 \%$ of patients were observed with graft edema which was statistically significant $(p=0.032)$. while $43.3 \%$ patients in control group showed discharge from STSGs site, this was insignificant in PRP group. $(p=0.022)$. Only $6.7 \%$ patients from PRP group required secondary grafting compared to $33.3 \%$ patients in control group who underwent secondary grafting due to graft loss, which was also statistically significant $(p=0.038)$.

$86.6 \%$ patients from case group needed first graft inspection on the 5 th day while $36.7 \%$ patients from control group needed first graft inspection within 5 days of procedure. $(p=0.037)$. A total of $66.7 \%$ patients from control group needed 3-5 times dressing whereas only $20 \%$ patients from case group needed 3-5 times dressing $(p=0.007)$.About $75 \%$ of the patients discharged after 10 days in control group, whereas $90 \%$ of patients were discharged within 10 post graft days in the PRP group. $(p=0.035)$

All the parameters used for the study showed statistically significant difference between the case and control group $(\mathrm{p}<0.05)$.

Table 1: Percentage distribution of age, gender, comorbidities, medication, location and etiology

\begin{tabular}{|l|l|l|}
\hline Demographic details & PRP group & Control group \\
\hline Age & $48.8 \pm 8.8$ & $52.1 \pm 10.9$ \\
\hline Male & $24,80 \%$ & $27,90 \%$ \\
\hline
\end{tabular}

\begin{tabular}{|c|c|c|}
\hline Female & $6,20 \%$ & $3,10 \%$ \\
\hline \multicolumn{3}{|l|}{ Location } \\
\hline Leg & $11,36.6 \%$ & $12,40 \%$ \\
\hline Foot & $10,33.3 \%$ & $8,26.7 \%$ \\
\hline Forearm & $5,16.7 \%$ & $4,13.3 \%$ \\
\hline Chest & $4,13.3 \%$ & $2,6.7 \%$ \\
\hline \begin{tabular}{|l|} 
Thigh \\
\end{tabular} & $0,0 \%$ & $3,10 \%$ \\
\hline \begin{tabular}{|l} 
Back \\
\end{tabular} & $0,0 \%$ & $1,3.3 \%$ \\
\hline \multicolumn{3}{|l|}{ Etiology } \\
\hline Necrotizing fasciitis & $13,43.3 \%$ & $10,33.3 \%$ \\
\hline Diabetic foot & $7,23.3 \%$ & $6,20 \%$ \\
\hline Cellulitis & $4,13.3 \%$ & $5,16.7 \%$ \\
\hline Burn & $3,10 \%$ & $3,10 \%$ \\
\hline Trauma & $2,6.67 \%$ & $3,10 \%$ \\
\hline Amputation & $1,3.3 \%$ & $3,10 \%$ \\
\hline \multicolumn{3}{|l|}{ Co-morbidities } \\
\hline Diabetes & $12,40 \%$ & $10.33 .3 \%$ \\
\hline Hypertension & $7,23.3 \%$ & $2,20 \%$ \\
\hline
\end{tabular}

Table 2: Outcome variables

\begin{tabular}{|c|c|c|c|c|c|}
\hline & \begin{tabular}{|l|} 
Instant \\
Adhesion
\end{tabular} & \begin{tabular}{|l|} 
Graft \\
Odema
\end{tabular} & \multicolumn{2}{|c|}{$\begin{array}{l}\text { Discharge from } \\
\text { Graft Site }\end{array}$} & \begin{tabular}{|l} 
Graft \\
Loss
\end{tabular} \\
\hline Case & $30,100 \%$ & $3,10 \%$ & \multicolumn{2}{|c|}{$4,13.3 \%$} & $2,6.7 \%$ \\
\hline Control & 0 & $11,36.6 \%$ & \multicolumn{2}{|c|}{$13,43.3 \%$} & $10,33.3 \%$ \\
\hline \multirow{2}{*}{\multicolumn{3}{|c|}{\begin{tabular}{|l|l|} 
p Value & 0.0001 \\
\end{tabular}}} & \multicolumn{2}{|l|}{0.022} & 0.038 \\
\hline & & & Case & \begin{tabular}{|l|l} 
Control & $\mathrm{F}$ \\
\end{tabular} & p Value \\
\hline \multicolumn{6}{|c|}{ Day of $1^{\text {st }}$ graft inspection } \\
\hline \multicolumn{3}{|c|}{$<5$ days } & 4 & 11 & \multirow[t]{2}{*}{0.037} \\
\hline$>5$ days & & & 26 & 19 & \\
\hline \multicolumn{6}{|c|}{ Frequency of dressings } \\
\hline 1-2 time & & & 24 & 10 & \multirow[t]{2}{*}{0.007} \\
\hline $3-5$ time & & & 6 & 20 & \\
\hline \multicolumn{6}{|c|}{ Stay in the hospital } \\
\hline \multicolumn{3}{|c|}{$<10$ days } & 22 & 14 & \multirow[t]{2}{*}{0.035} \\
\hline \multicolumn{3}{|c|}{$>10$ days } & 8 & 16 & \\
\hline
\end{tabular}

Results of assessment parameters

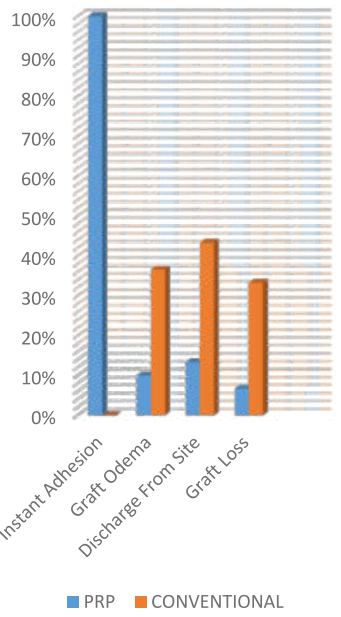

\section{GRAPH 1}

\section{DISCUSSION}

- PRP is a biological product defined as a portion of the plasma fraction of autologous blood with a platelet concentration above the baseline

- Platelet-Rich Plasma (PRP) is also known as Platelet-rich Growth Factors (GFs), Platelet-Rich Fibrin (PRF) matrix, PRF, and platelet concentrate.

- The concept and description of PRP started in the field $f$ hematology. Hematologists created the term PRP in the 1970s in order to describe the plasma with a platelet count above that of peripheral blood 
- It was initially used as a transfusion product to treat patients with thrombocytopenia.

- Subsequently, PRP has been used predominantly in the musculoskeletal field in sports injuries. Other medical fields that also use PRP are cardiac surgery, pediatric surgery, gynecology, urology, plastic surgery, and ophthalmology.

- More recently, the interest in the application of PRP in dermatology i.e., in tissue regeneration, wound healing, scar revision, skin rejuvenating effects, and alopecia, has increased.

- In this study the primary aim of application of PRP prior to resurfacing with skin graft was to facilitate its instant stable adhesion as result of its cohesive and adhesive nature to the wound bed without mechanical fixation.

- It also facilitated more rapid soft-tissue wound healing and faster vascularization of the healing tissue by delivering growth factors.

- We also achieved other benefits like hemostasis, reduction in operating time and frequency of postoperative dressings.

- PRP exerts its beneficial effects via the degranulation of the alpha granules in platelets that contain growth factors. The active secretion of these growth factors begins within minutes of the start of the coagulation sequence, and more than $90 \%$ are secreted during the first hour.

- Growth factors serve to accelerate the wound-healing process by increasing cellular proliferation, matrix formation, connective tissue healing, angiogenesis, and collagen synthesis.

- PRP functions as a tissue sealant and drug delivery system, with the platelets initiating wound repair by releasing locally acting growth factors via $\square$-granules degranulation.

- These growth factors aid healing by attracting undifferentiated cells in the newly formed matrix and triggering cell division.

- PRP may suppress cytokine release and limit inflammation, interacting with macrophages to improve tissue healing and regeneration, promote new capillary growth, and accelerate epithelialization in chronic wounds.

- In our study there was instant adherence of skin graft to wound bed in all 30 patients in the PRP test group as compared to control group in whom it did not happened.

\section{CONCLUSION}

The use PRP is safe and effective in the management of acute wounds.

It is a cost-effective procedure, helps in early skin grafting, INCREASED GRAFT ACCEPTANCE and reduced hospital stay.

It is found to be highly beneficial in many aspects both to the patient and surgeon based on our results.

We recommend the use of autologous PRP routinely in all age groups and all types of wounds prior to resurfacing to ensure better and faster healing as suggested by our results.

\section{REFERENCES}

1. Smith RG, Gassmann CJ, Campbell MS. Plateletrich plasma: properties and clinical applications. J Lancaster Gen Hosp. 2007:2(2):73-7.

2. Everts PA, Knape JT, Weibrich G, Schönberger JP, Hoffmann J, Overdevest EP, et al. Platelet-rich plasma and platelet gel: a review. J Extra-corporeal Tech. 2006;38(2):174

3. Schade VL, Roukis TS. Use of platelet-rich plasma with split-thickness skin grafts in the high-risk patient. Foot Ankle Specialist. 2008;1(3):155-9.

4. Alves R, Grimalt R. A review of platelet-rich plasma: history, biology, mechanism of action, and classification. Skin Appendage Dis. 2018;4:18-24.

5. Lacci KM, Dardik A. Platelet-rich plasma: support for its use in wound healing. Yale JBio Med. 2010;83(1):1-9.

6. Eppley BL, Woodell JE, Higgins J. Platelet quantification and growth factor analysis from platelet-rich plasma: implications for wound healing. Plastic Reconstructive Surg. 2004;114(6):1502-8
7. Tischler M. Platelet rich plasma: the use of autologous growth factors to enhance bone and soft tissue grafts NY State Dental J. 2002;68(3):22

8. Steed DL, Goslen JB, Holloway GA, Malone JM, Bunt TJ, Webster MW. Randomized prospective double-blind trial in healing chronic diabetic foo ulcers: CT-102 activated platelet supernatant, topical versus placebo. Diab Care. 1992; 15(11):1598-604

9. Cervelli V, Gentile P, Grimaldi M. Regenerative surgery: use of fat grafting combined with plateletrich plasma for chronic lower-extremity ulcers. Aesth Plastic Surg. 2009;33(3):340.

10. Bielecki TM, Gazdzik TS, Arendt J, Szczepanski T, Krol W, Wielkoszynski T. Antibacterial effect of autologous platelet gel enriched with growth factors and other active substances: an in vitro study. J Bone Joint Surg. 2007;89(3):417-20

11. Foster K, Greenhalgh D, Gamelli RL, Mozingo D, Gibran N, Neumeister M, et al. Efficacy and safety of a fibrin sealant for adherence of autologous skin grafts to burn wounds: results of a phase 3 clinical study. J Burn Care Res. 2008;29(2):293-303.

12. Gibran N, Luterman A, Herndon D, Lozano D, Greenhalgh DG, Grubbs L, et al. Comparison of fibrin sealant and staples for attaching splitthickness autologous sheet grafts in patients with deep partial-or full-thickness burn wounds: $\alpha$ phase 1/2 clinical study. J Burn Care Res. 2007:28:401-8

13. Ratajczak J, Vangansewinkel T, Gervois P, Merckx G, Hilkens P, Quirynen M, et al. Angiogenic properties of 'leukocyte-and platelet-rich fibrin'. Sci Rep. 2018;8(1):14632.

14. Sommeling CE, Heyneman A, Hoeksema H, Verbelen J, Stillaert FB, Monstrey $\mathrm{S}$. The use of platelet-rich plasma in plastic surgery: a systematic review. J Plastic Reconstructive Aesth Surg. 2013;66(3):301-11.

15. EL Mabood, Ali HE. Platelet rich plasma vs. conventional dressing: does this really affect diabetic foot wound-healing outcomes?. Egypt J Surg 2018;37(1):16

16. Prabhu R, Vijayakumar C, Chandra AA, Balagurunathan $\mathrm{K}$, Kalaiarasi $\mathrm{R}$, Venkatesan $\mathrm{K}$, et al. Efficacy of homologous, platelet-rich plasma dressing in chronic non-healing ulcers: an observational study. Cureus. 2018;10(2). 